\title{
Transient Spinal Cord Ischemia as Presenting Manifestation of Polycythemia Vera
}

\author{
Sónia Costa ${ }^{a} \quad J o a n a$ Marques $^{c} \quad$ Anabela Barradas $^{b}$ \\ Ana Valverde \\ ${ }^{a}$ Neurology Department and ${ }^{b}$ Immunochemotherapy Department, Hospital \\ Professor Dr. Fernando Fonseca EPE, Amadora, and 'Neurology Department, \\ Instituto Português de Oncologia Francisco Gentil, Lisbon, Portugal
}

\section{Key Words}

Spinal cord ischemia - Polycythemia vera - JAK2 V617F mutation

\begin{abstract}
Spinal arterial vascularization is supplied by a large anastomotic net, making spinal ischemic events far less common than ischemic cerebral strokes. Polycythemia vera, due to blood hyperviscosity and activated platelet aggregation, is associated with a higher risk of arterial and venous thrombotic events. We report a patient with spinal cord transient ischemic attacks, a rarely presenting manifestation, and polycythemia vera, which highlights the thrombotic potential of this disease, and the requirement of exhaustive diagnostic workout of a spinal ischemic event.
\end{abstract}

\section{Introduction}

Spinal arterial vascularization is supplied by a large anastomotic net, making spinal ischemic events far less common than ischemic cerebral strokes. Preobrashenski was the first to describe the anterior spinal artery syndrome in 1904, following Bastian's suggestion in 1882 that spinal cord softening might be the result of vascular occlusion [1]. Usually the causes of spinal ischemic events are: aortic or vertebral artery disease, resulting from atherosclerosis, embolic infarction or dissecting aneurism; compression/trauma; iatrogenic, following a number of surgical and diagnostic procedures; and less frequently, systemic diseases such as vasculitis or severe systemic hypotension. Prognosis of spinal cord infarct has not been completely determined, since, given its low frequency, the number of cases have been too small to define broadly the natural history of the disease [2]. 
We describe a case of transient spinal cord ischemia as a presenting manifestation of polycythemia vera, which is a rare hematologic disease associated with blood hyperviscosity and higher risk of thrombotic events.

\section{Case Report}

A 52-year-old male, Caucasian, with previous nephrolithiasis and a transient lower limbs motor deficit without medical investigation 12 years ago, presented, after physical effort, an acute low back pain without irradiation, with motor deficit in the lower limbs with no sensibility or sphincterian dysfunction. Initial neurologic examination disclosed proximal paraparesis with abolished myotatic and plantar reflexes, bilaterally. Spontaneous clinical recovery was observed, and $24 \mathrm{~h}$ later, only left lower limb hyperactive myotatic reflexes were present. Five months later, a new episode with identical symptoms occurred, resolving in $30 \mathrm{~min}$, with a normal neurologic examination on arrival at emergency room.

Initial laboratory evaluation showed only high erythrocyte and platelet counts, with increased hemoglobin and hematocrit. Posterior exhaustive laboratory investigation excluded vasculitis, collagen and coagulation disorders and finally infectious diseases such as syphilis, human immunodeficiency virus and Epstein-Barr virus (table 1). The thoracic-abdomino-pelvic CT (with angio CT) scan showed a splenomegaly with no signs of aortic dissection. Lumbar puncture, brain CT scan, spinal MRI, somatosensitive evoked potentials and electromyogram were normal. A spinal angiography was performed, showing artery of Adamkiewicz origin at the T11 level, with no ascending branch (fig. 1).

Diagnosis of polycythemia vera was confirmed after bone marrow biopsy and myelogram, low levels of erythropoietin and positive JAK2 V617F mutation. He was treated with phlebotomies, first biweekly then monthly, hydroxyurea and a platelet inhibitor. In a follow-up time of two years, no new neurologic symptoms relapsed since treatment was started.

\section{Discussion}

Polycythemia vera is a chronic myeloproliferative neoplasm, characterized by overproduction of mature blood elements with predominance of erythroid lineage, variable degree of bone marrow fibrosis and extramedullary hematopoiesis (in spleen and liver) typical of more advanced phases of the disease. In 2005 the first molecular abnormality, represented by a point mutation in JAK2 exon 14, was identified, leading to revision of WHO diagnostic criteria in 2008 [3]. This mutation is present in $95 \%$ of cases [4].

This disease is associated with a higher risk of arterial and venous thrombotic events, namely in the cerebral, ocular, coronary and pulmonary territories. In a large prospective cohort of 1,638 patients with polycythemia vera, the incidence of recurrent thrombosis was $5 \%$ patient-years among individuals $<65$ years old and $10.9 \%$ patient-years among those $>65$ years old [5]. In a recent survey from Italian GINEMA group, the calculated recurrence rate is $5.6 \%$ per patient-year, and the cumulative probability is $49.9 \%$ at 10 years [6]. The first recurrent thrombosis involved arterial vessels in $60.8 \%$ of cases and venous vessels in $39.7 \%$ of cases. The overall probability of recurrence was not predicted by whether the first manifestation was in the arterial or venous district, but re-thrombosis occurred preferentially in the same vascular district previously affected $[5,6]$.

Nevertheless, the mechanisms finally responsible for the increased thrombotic tendency have not been clearly elucidated, although risk factors for thrombosis have been identified and are currently employed for stratifying patients to appropriate therapeutic 
options. The two most important thrombotic risk factors are advanced age ( $>60$ years) and prior history of thrombosis [6]. Abnormalities of blood cells, activation of neutrophils and platelets, and a hypercoagulability state, can all act in conjunction to lead to thrombosis $[4,7]$. Intriguing data also point to the JAK2 V617F mutation as both a marker and a mechanism for thrombosis, besides a diagnostic tool, further raising the risk of thrombosis in younger patients [4].

Current treatment of this disease is mainly aimed at preventing major cardiovascular events and is based on the risk category to which the patient belongs. For low-risk patients ( $<60$ years old and no previous thrombosis), phlebotomies and platelet inhibitors, whereas for high-risk patients ( $>60$ years old and/or previous thrombosis), cytotoxic therapy should also be instituted.

We report a patient with spinal cord transient ischemic attacks, a rarely presenting manifestation, which highlights the thrombotic potential of this disease. The anatomy of spinal cord blood supply is complicated by individual variations in the number, size and location of its vasculature [8]. However, certain features are relatively consistent and of clinical importance. There are 31 pairs of radicular arteries that enter the vertebral canal through the intervertebral foramen, but the majority of these arteries regress during development, leaving only four to eight contributors, termed radiculomedullary arteries. The largest and most constant radicular artery is the arteria radicularis magna or artery of Adamkiewicz, which usually arises from level T9 to T12. The midthoracic region has fewer arteries per $\mathrm{cm}$ of spinal cord than any other spinal cord region, making this region particularly vulnerable to ischemia during global hypoperfusion, especially T4 to T8 spinal cord levels.

The origin of artery of Adamkiewicz at T11 level, with no ascending branch, as seen in our patient's spinal angiography, was interpreted as an anatomical variation that predisposed to several spinal cord transient attacks. Since he was a high-risk patient because of recurrent thrombosis, cytotoxic therapy besides platelet inhibitors and phlebotomies was instituted, with good clinical response. Recently, patients carrying acquired JAK2 V617F mutation, as this patient, have been demonstrated to obtain better cytoreduction and have a lower rate of arterial thrombosis when treated with hydroxyurea [9].

In conclusion, this case report supports the knowledge that acute nontraumatic complete or partial transverse myelopathies as well as classic vascular spinal cord syndromes are etiologically ambiguous and require well-established diagnostic procedures such as exhaustive blood evaluation, lumbar puncture, neuroimaging as spinal MRI, as well as thoracic-abdomino-pelvic CT (with angio CT), and eventually a spinal angiography $[10,11]$. Polycythemia vera, although a rare hematological disease, has to be considered as a possible cause of ischemic events in cerebral or spinal territories, when other more frequent etiologies are excluded.

Finally, this case holds up JAK2 mutation as a novel disease-associated risk factor, useful for predicting cardiovascular complications of polycythemia vera [12]. 
Table 1. Laboratory evaluation

\begin{tabular}{|c|c|c|c|}
\hline Blood & $\begin{array}{l}\text { Results at } \\
\text { baseline }\end{array}$ & $\begin{array}{l}\text { Results after } \\
6 \text { months of } \\
\text { treatment }\end{array}$ & $\begin{array}{l}\text { Reference } \\
\text { values }\end{array}$ \\
\hline $\mathrm{Hg}, \mathrm{g} / \mathrm{dl}$ & $20.1 \uparrow$ & 17.4 & $13-18$ \\
\hline Hematocrit, \% & $59.3 \uparrow$ & 53.3 & $40-54$ \\
\hline Leukocytes, $\mu \mathrm{l}$ & 9,100 & 7,800 & $4,000-11,000$ \\
\hline Platelets, $\mu \mathrm{l}$ & $613,000 \uparrow$ & $570,000 \uparrow$ & $150-400,000$ \\
\hline ESR, mm & 1 & 1 & $<13$ \\
\hline $\mathrm{CRP}, \mathrm{mg} / \mathrm{dl}$ & 0.42 & 0.07 & $<1$ \\
\hline ANA/Anti DNA ds & Neg/Neg & - & - \\
\hline Anti SSA/SSB & Neg/Neg & - & - \\
\hline HIV 1 e $2 / V D R L$ & Neg & - & - \\
\hline$\beta 2$-Microglobulin, mg/l & 2.28 & 1.31 & $0.67-1.3$ \\
\hline Erythropoietin, mU/l & $1.52 \downarrow$ & - & $2.6-34$ \\
\hline
\end{tabular}

$\mathrm{Hg}=$ Hemoglobin; $\mathrm{ESR}=$ erythrocyte sedimentation rate; $\mathrm{CRP}=\mathrm{C}$ reactive protein.

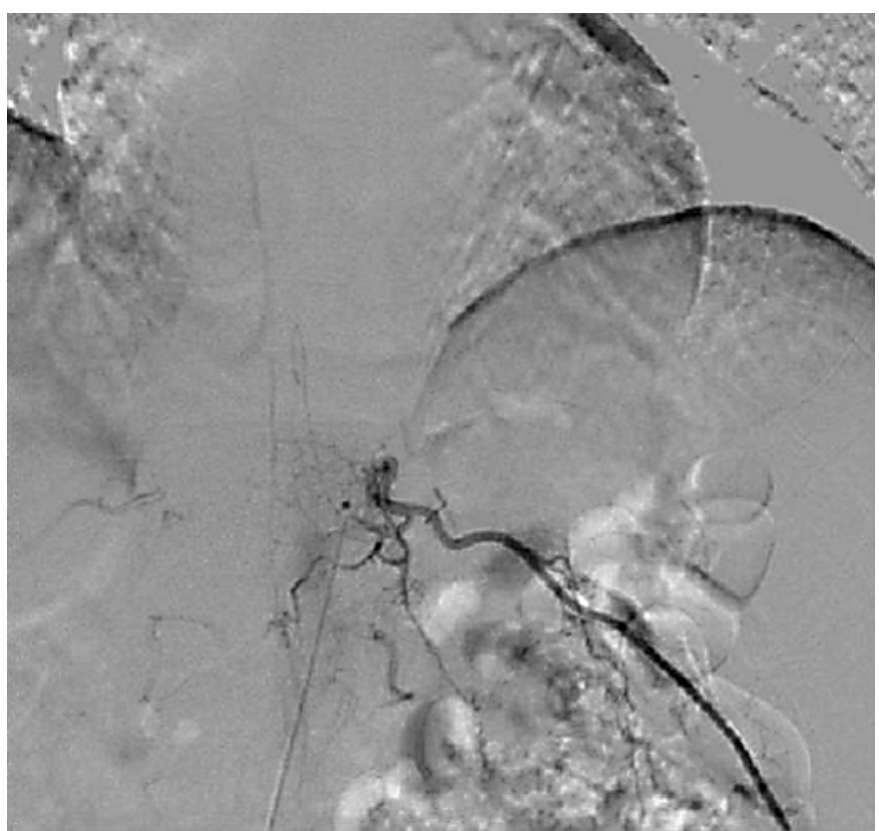

Fig. 1. Spinal angiography showed origin of artery of Adamkiewicz at the T11 level, with no ascending branch. 


\section{References}

$\checkmark 1$ Cheshire WP, Santos C, Massey EW, Howard J Jr: Spinal cord infarction: etiology and outcome. Neurology 1996;47:321-333.

-2 Salvador de la Barrera S, Barca-Buyo A, Montoto-Marqués A, Ferreiro-Velasco M, Cidoncha-Dans M, Rodriguez-Sotinho A: Spinal cord infarction and recovery in a series of 36 patients. Spinal Cord 2001;39:520525 .

-3 Tefferi A, Thiele J, Orazi A, et al: Proposals and rationale for revision of the World Health Organization diagnostic criteria for polycythemia vera, essential thrombocythemia, and primary myelofibrosis: recommendations from an ad hoc international expert panel. Blood 2007;110:1092-1097.

4 Vannucchi AM: Insights into the pathogenesis and management of thrombosis in polycythemia vera and essential thrombocythemia. Intern Emerg Med 2010;5:177-184.

5 Marchioli R, Finazzi G, Landolfi R, et al: Vascular and neoplastic risk in a large cohort of patients with polycythemia vera. J clin oncol 2005;23:2224-2232.

-6 De Stefano V, Za T, Rossi E, Vannucchi AM, et al; GIMEMA CMD-Working Party: Recurrent thrombosis in patients with polycythemia vera and essential thrombocythemia: incidence, risk factors, and effect of treatments. Haematologica 2008;93:372-380.

7 Kwaan HC, Wang J: Hyperviscosity in polycythemia vera and other red cell abnormalities. Semin Thromb Hemost 2003;29:451-458.

-8 Sanson TA, Friedman JH: Spinal cord infarction. Report of 8 cases and review of the literature. Medicine (Baltimore) 1989;68:282-292.

9 Campbell PJ, Scott LM, Buck G, et al: Definition of subtypes of essential thrombocythaemia and relation to polycythaemia vera based on JAK2 V617F mutation status: a prospective study. Lancet 2005;366:1945-1953.

10 Martinelli V, Comi G, Rovaris M, et al: Acute myelopathy of unknown aetiology: a clinical, neurophysiological and MRI study of short- and long-term prognostic factors. J Neurol 1995;242:497-503.

11 Caplan LR: Spinal-cord strokes; in Caplan LR (ed): Stroke: A Clinical Approach. 2nd ed. Boston, ButterworthHeinemann, 1993, pp 487-496.

12 Vannucchi AM: JAK2 Mutation and thrombosis in the myeloproliferative neoplasms. Curr Hematol Malig Rep 2010;5:22-28. 